Western Washington University Western CEDAR

$9-1989$

\title{
Definitizable Extensions of Positive Symmetric Operators in a Krein Space
}

Branko Ćurgus

Western Washington University, branko.curgus@wwu.edu

Follow this and additional works at: https://cedar.wwu.edu/math_facpubs

Part of the Mathematics Commons

\section{Recommended Citation}

Curgus, Branko, "Definitizable Extensions of Positive Symmetric Operators in a Krein Space" (1989). Mathematics. 74. https://cedar.wwu.edu/math_facpubs/74

This Article is brought to you for free and open access by the College of Science and Engineering at Western CEDAR. It has been accepted for inclusion in Mathematics by an authorized administrator of Western CEDAR. For more information, please contact westerncedar@wwu.edu. 
Integra1 Equations and Operator Theory Vol. 12 (1989)
$0378-620 X / 89 / 050615-17 \$ 1.50+0.20 / 0$

(c) 1989 Birkhäuser Verlag, Base1

DEFINITIZABLE EXTENSIONS OF POSITIVE SYMMETRIC OPERATORS IN A KREIN SPACE

\author{
Branko Ćurgus
}

The Friedrichs extension and the Krein extension of a positive operator in a Krein space are characterized in terms of their spectral functions in a Krein space.

\title{
INTRODUCTION
}

In the fundamental paper $[\mathrm{Kr}], \mathrm{M}$. G. Krein developed the theory of selfadjoint extensions of semibounded symmetric (densely defined) operators in a Hilbert space. In particular he constructed all positive selfadjoint extensions of a given positive symmetric operator and he proved among other things that if the set of all positive selfadjoint operators is equipped with a natural partial ordering (as defined in [Ka, VI-\$2.5]) then a greatest and a smallest positive selfadjoint extension exist. In [Kr] the greatest extension is called "hard" and it was shown to coincide with the Friedrichs extension. The smallest extension, that is, the "soft" extension in the terminology of krein, we call the Krein extension (see [AS]). This theory was completed by Birman [Bi] and Vishik [V], and was also discussed in [AN], $[F],[A S],[S]$. A similar problem for positive symmetric subspaces (multivalued operators) was studied in [CS].

In this note we consider definitizable extensions of a given semibounded symmetric operator in a Krein space. Positive definitizable extensions of a positive operator in a Krein space were first studied in [LI] in order to describe all the general- 
ized resolvents of a positive operator with equal and finite defect numbers. In section 2, a characterization of the Friedrichs and the Krein extensions in a krein space is formulated in terms of their spectral functions. These results are analogous to the results of Krein which we quote in the first section of this note. The characterization of the form domain of a definitizable operator, stated in section 3, can be used in order to prove uniform convergence of eigenfunction expansions associated with ordinary differential operators having an indefinite weight function. These results will be published elsewhere. An equivalent description of the form domain of a positive definitizable operator appears in [JL, Proposition 3.1].

The author is indebted to Professor Heinz Langer for helpful suggestions and continual interest in this work.

\section{PRELIMINARIES}

Let $(H,(.)$,$) be a Hilbert space with the norm \|f\|$ $=(f, f)^{1 / 2}(f \in H)$. All operators which we consider in this note are supposed to be linear and densely defined. We denote by $D(S)$ the domain of $S$, by $R(S)$ the range of $S$, i.e., $R(S)$ $=S(D(S))$, and by $S^{*}$ the adjoint of $S$ in $(H,(.)$,$) . An$ operator $S$ is called symmetric (selfadjoint) in $(H,(.,$.$) ) if$ $S \subset S^{*}\left(S=S^{*}\right.$, resp.). A closed symmetric operator $S$ is said to be bounded from below in $(H,(.)$,$) if$

$$
(\mathrm{Sf}, \mathrm{f}) \geq \alpha(f, f) \quad(f \in D(S))
$$

for some $\alpha \in \mathbb{R}$. The operator $S$ is called positive (strictly positive) if $\alpha \geq 0(\alpha>0$, resp. $)$.

Throughout this section we suppose that the operator $S$ is bounded from below in $(H,(.)$,$) .$

Let $D[S]$ be the domain of the closed symmetric form constructed from the operator $S$ in $(\mathbf{H},(, \ldots))$. Recall that $D$ [S] consists of all $f \in H$ for which there exists a sequence $\left(\phi_{\mathrm{n}}\right) \subset \mathrm{D}(\mathrm{S})$ such that $\phi_{\mathrm{n}} \rightarrow \mathrm{f}$ in $\mathrm{H}$ and 
Ćurgus

$$
\left(\mathrm{S}\left(\phi_{\mathrm{n}}-\phi_{\mathrm{m}}\right), \phi_{\mathrm{n}}-\phi_{\mathrm{m}}\right) \rightarrow 0 \quad(\mathrm{n}, \mathrm{m} \rightarrow+\infty)
$$

(see $[\mathrm{Ka}, \mathrm{VI}-\S 1.4]$ ). For a selfadjoint operator $S$ in the Hilbert space $(H,(.)$,$) by \mathbf{D}[S] \subset \mathbf{H}$ we denote the completion of $D(S)$ with respect to the norm $\left\|(|S|+I)^{1 / 2}.\right\|$. For a bounded from below selfadjoint operator $s$ these two definitions of $D[S]$ coincide (see $[C])$. We have $D[S]=D\left(|S|^{1 / 2}\right)$.

By $S_{F}$ we denote the Friedrichs extension of $S$ in $(H,(.,)$.$) (see [\mathrm{Ka}],[\mathrm{W}])$. From the definition of $S_{F}$ it follows that $\mathrm{D}\left[\mathrm{S}_{\mathrm{F}}\right]=\mathrm{D}[\mathrm{S}]$. Put $\mathrm{N}_{\mathrm{a}}=\operatorname{ker}\left(\mathrm{S}^{*}-\mathrm{aI}\right)$ for a $\epsilon \phi$. The next theorem follows from $[\mathrm{Kr}]$.

THEOREM 1.1. Let $S_{1}$ be a selfadjoint extension of $S$ and let $F$ denote the spectral function of $S_{1}$. Then the following statements are equivalent:

(i) $s_{I}=s_{F}$.

(ii) $D\left(S_{1}\right)=D\left(S^{*}\right) \cap D[S]$.

(iii) $\mathbf{D}\left[S_{1}\right]=D[S]$.

(iv) For some (and hence for all) a $\epsilon \varrho\left(s_{1}\right)$ we have

$$
\mathrm{N}_{\mathrm{a}} \cap \mathbb{D}\left[\mathrm{S}_{1}\right]=\{0\} .
$$

(v) For some (and hence for all) a $\epsilon \varrho\left(S_{1}\right)$ and for all $\phi \in \mathbf{N}_{\mathrm{a}}$ we have

$$
\int_{\mathbb{R}} t d\left(F_{t} \phi, \phi\right)=+\infty .
$$

In order to state an analogous theorem for the krein extension $S_{K}$ of a positive operator $S$ in $(H,(.)$,$) we de-$ fine the closure $R[S]$, of the range of $S$ with respect to the symmetric form constructed from $S$ in $(H,(.,)$.$) , to be the set$ of all $f \in H$ for which there exists a sequence $\left(\phi_{n}\right) \subset D(S)$ such that $S \phi_{\mathrm{n}} \rightarrow f$ in $H$ and

$$
\left(S\left(\phi_{\mathrm{n}}-\phi_{\mathrm{m}}\right), \phi_{\mathrm{n}}-\phi_{\mathrm{m}}\right) \rightarrow 0 \quad(\mathrm{n}, \mathrm{m} \rightarrow+\infty) .
$$

LEMMA 1.2. If $S$ is a positive selfadjoint operator in $(H,(., \cdot))$ then $R[S]=R\left(S^{1 / 2}\right)$.

PROOF. Let $L$ be the closure of $R(S)$ in $\mathbf{H}$. Then 
the restriction $\mathrm{S} / \mathrm{L}$ is a positive selfadjoint operator in the Hilbert space $(L,(.)$,$) and zero is not an eigenvalue of S \mid L$. The operator $(S \mid L)^{-1}$ is defined on $R(S)$ and $D\left[(S \mid L)^{-1}\right]$ consists of all $f \in I$ for which there exists a sequence $\left(\eta_{\mathrm{n}}\right) \subset \mathbf{R}(\mathrm{S})$ such that $\eta_{\mathrm{n}} \rightarrow \mathrm{f}$ in $\mathrm{L}$ (i.e. in $\mathbf{H}$ ) and

$$
\left((\mathrm{S} \mid \mathrm{L})^{-1}\left(\eta_{\mathrm{n}}-\eta_{\mathrm{m}}\right), \eta_{\mathrm{n}}-\eta_{\mathrm{m}}\right) \rightarrow 0(\mathrm{n}, \mathrm{m} \rightarrow+\infty) \text {. }
$$

Let $\eta_{\mathrm{n}}=\mathrm{S} \phi_{\mathrm{n}}, \mathrm{n}=1,2, \ldots$, with some $\phi_{\mathrm{n}} \in \mathrm{L} n \mathrm{D}(\mathrm{S})$. Then $\mathrm{S} \phi_{\mathrm{n}} \rightarrow \mathrm{f}$ in $\mathrm{H}$ and $\left(\phi_{\mathrm{n}}-\phi_{\mathrm{m}}, \mathrm{S}\left(\phi_{\mathrm{n}}-\phi_{\mathrm{m}}\right)\right) \rightarrow 0(\mathrm{n}, \mathrm{m} \rightarrow+\infty)$. Hence $\mathrm{D}\left[(\mathrm{S} \mid \mathrm{L})^{-1}\right]=\mathbf{R}[\mathrm{S}]$. Since $\mathrm{D}\left[(\mathrm{S} \mid \mathrm{L})^{-1}\right]=\mathrm{D}\left((\mathrm{S} \mid \mathrm{L})^{-1 / 2}\right)=\mathrm{R}\left(\mathrm{S}^{1 / 2}\right)$, the lemma is proved.

The following theorem collects some results from [ $\mathrm{Kr}]$ and [AN].

THEOREM 1.3. Let $S$ be a positive operator in $(H,(.,)$.$) and let S_{1}$ be a positive selfadjoint extension of $S$. Denote by $F$ the spectral function of $S_{1}$. The following statements are equivalent:
(i) $S_{1}=s_{K}$.
(ii) $R\left(S_{1}\right)=R\left(S^{*}\right) \cap R[S]$.
(i $) \quad D\left(S_{1}\right)=\left\{f \in H: S^{*} f \in R[S]\right\}$.
(iii) $R\left[S_{1}\right]=R[S]$.
(iv) For some (and hence for all) a $\epsilon \varrho\left(s_{1}\right)$ we have

$$
\mathbf{N}_{\mathrm{a}} \cap \mathbf{R}\left[\mathrm{S}_{1}\right]=\{0\} .
$$

(v) For some (and hence for all) a $\epsilon \rho\left(S_{1}\right)$ and for all $\phi \epsilon \mathbf{N}_{\mathrm{a}}$ we have

$$
\int_{\mathbb{R}} 1 / t d\left(F_{t} \phi, \phi\right)=+\infty
$$

PROOF. The equivalence $(i) \Leftrightarrow\left(i_{1}\right)$ is corollary 2 in [AN]. The implication $\left(i_{1}\right)=>(i i)$ is obvious. If we suppose (ii), then we have $\operatorname{ker}\left(S_{1}\right)=R\left(S_{1}\right)(\perp)=\left(R\left(S^{*}\right) \cap \mathbf{R}[S]\right)^{(\perp)}=N_{0}$, where $(1)$ denotes the orthogonal complement in the Hilbert space. Now $\left(i_{1}\right)$ follows easily. From the definition of the set $R\left[S_{1}\right]$ it follows that (ii) implies (iii). If we assume (iii), then $R\left(S_{1}\right) \cap R\left(S^{*}\right) \subset R[S]=R\left(S_{K}\right)$. Let $f \in D\left(S_{1}\right)$ and let $g \in D\left(S_{K}\right)$ be such that $S_{1} f=S_{K} g$. Then $f-g \in N_{O} \subset D\left(S_{K}\right)$. 
It follows that $f \in D\left(S_{K}\right)$. Hence $D\left(S_{1}\right) \subset D\left(S_{K}\right)$, and consequently $S_{1}=S_{K}$. Thus $(i i i) \Rightarrow(i)$. The equivalence (iv) $\Leftrightarrow(v)$ is obvious and $(i)<=>(v)$ is proved in Theorem 8 in [Kr] (see also Theorem 4.1 in $[S]$ ) for $a=-1$.

In order to prove that (iv) is equivalent to $N_{-1} \cap R\left[S_{1}\right]$ $=\{0\}$, we observe that we can suppose $a \neq 0$ in (iv). Otherwise, if $a=0$, (iv) implies $N_{0}=\{0\}$, and therefore $s$ is essentially selfadjoint. Our theorem is trivial in this case. Further, observe that (iv) implies $\mathbf{N}_{0} \subset \mathrm{D}\left(\mathrm{S}_{1}\right)$. Indeed, $\mathbf{N}_{0} \subset \mathbf{D}\left(\mathrm{S}^{*}\right)=\mathrm{D}\left(\mathrm{S}_{1}\right)+\mathbf{N}_{\mathrm{a}}$ and, hence, for $f \in \mathbf{N}_{0}$ we have $f=g+h$, with $g \in D\left(S_{1}\right), h \in N_{a}$. It follows that $0=S^{*} f$ $=s_{1} g+a h$, i.e., $S_{1} g=-a h \in N_{a} \cap R\left(S_{1}\right)=\{0\}$. Since $a \neq 0$, we have $h=0$. Thus $f=g \in D\left(S_{I}\right)$, i.e., $N_{0} \subset D\left(S_{1}\right)$. Suppose that (iv) holds and let $f \epsilon \mathbf{N}_{-1} \cap R_{\left[S_{1}\right]}$. Then $f \epsilon R\left(S^{*}\right)$ $=R\left(S_{1}\right)+N_{a}$, i.e., $f=u+v$, with $u \in R\left(S_{1}\right), v \epsilon N_{a}$. It follows that $v=f-u \in R\left[S_{1}\right] \cap N_{a}=\{0\}$. Hence $f=u \in R\left(S_{1}\right)$. Let $f=S_{1} w$, with $w \in D\left(S_{1}\right)$. Then $S^{*} f=-f=-S_{1} w$ implies $f+w \in N_{O} \cap D\left(S_{1}\right)$. Thus $f \in D\left(S_{1}\right)$. since $S_{1} f=s^{*} f=-f$ and $-1 \in \varrho\left(S_{1}\right)$, it follows that $f=0$. The converse implication can be proved in the same manner.

\section{THE FRIEDRICHS AND THE KREIN EXTENSIONS IN A KREIN SPACE}

Let $(\pi,[.,]$.$) be a Krein space (see [B o],[A I]), J$ a fundamental symmetry on $K,(f, g)=[J f, g](f, g \in K)$ and $\|\cdot\|$ the corresponding norm on the Hilbert space $(\mathrm{K},(.,)$.$) . All$ topological notions in the Krein space $k$ are understood with respect to the topology generated by $\|\cdot\|$. This topology is independent of the choice of the fundamental symmetry $\mathrm{J}$. For this and other facts about Krein spaces see [Bo] or [AI]. By $A^{+}$ we denote the adjoint of an operator $A$ in the Krein space $(K,[.,]$.$) . If B=J A$ and $S=A J$, then $A^{+}=J B^{*}=S^{*} J$, where * denotes operator adjoints in the Hilbert space $((K,(.)$,$) . An operator A$ is said to be symetric (selfadjoint) in the Krein space $(K,[.,]$.$) if A \subset A^{+}\left(A=A^{+}\right.$, resp.). A closed symmetric operator $A$ is said to be bounded 
from below in a krein space $(\mathrm{K},[.,]$.$) if$

$$
[A f, f] \geq \alpha\|f\|^{2} \quad(f \in D(A))
$$

for some $\alpha \in \mathbb{R}$. The operator $A$ is called positive (strictly positive) if $\alpha \geq 0$ ( $\alpha>0$, resp.). Obviously, an operator A has one of the above defined properties in the krein space if and only if the operators $B=J A$ and $S=A J$ have the corresponding property in the Hilbert space $(K,(.)$,$) . We use the common$ definition of definitizable operators in a Krein space, see [L2]. $A$ definitizable operator $A$ in a krein space has a spectral function, possibly with critical points on the real axis (see [J], [L2], [AI]). The set of all critical points of the spectral function, which are also called the critical points of the definitizable operator $A$, will be denoted by $C(A)$.

Throughout this note we suppose that the operator A is bounded from below in the Krein space $\mathbf{K}$. Hence the operator $J A=B$ is bounded from below in the Hilbert space $(K,(.,)$.$) .$ For such $A$ we consider the set $D[J A]$ defined in the Hilbert space $(R,(\cdot)$,$) . The set D[J A]$ is the domain of the closure of the sesquilinear form [A.,.] and it does not depend on the choice of the fundamental symmetry $J$ (see [C]).

Let $B_{F}$ be the Friedrichs extension of $B=J A$ in the Hilbert space $(K,(.)$,$) . The operator A_{F}:=J_{B} F$ is said to be the Friedrichs extension of $A$ in the Krein space $K$. By $\mathbf{M}_{\mathrm{a}}$ we denote the kernel of $A^{+}-a I, a \epsilon \phi$. The following theorem is analogous to Theorem 1.1 . THEOREM 2.1. Let $A_{1}$ be a definitizable extension of $A$ in $(K,[.,]$.$) and let E$ denote the spectral function of $A_{1}$. Then the following statements are equivalent:

(i) $A_{1}=A_{F}$.

(ii) $D\left(A_{1}\right)=D\left(A^{+}\right) \cap D[J A]$.

(iii) $\mathrm{D}\left[\mathrm{JA}_{1}\right]=\mathrm{D}[\mathrm{JA}]$.

(iv) For some (and hence for all) a $\epsilon \varrho\left(A_{1}\right)$ we have

$$
\mathrm{M}_{\mathrm{a}} \cap \mathrm{D}\left[\mathrm{JA}_{1}\right]=\{0\} .
$$

(v) For some (and hence for all) a $\in Q\left(A_{1}\right)$ and for 
$\underline{\text { al1 }} \phi \in \mathbf{M}_{\mathrm{a}}$ we have

$$
\left|\int_{\Omega_{\infty}} t \mathrm{~d}\left[\mathrm{E}_{t} \phi, \phi\right]\right|=+\infty .
$$

Here $\Omega_{\infty}$ is an arbitrary closed neighborhood of $\infty$ in $\overrightarrow{\mathbb{R}}$, such that $\Omega_{\infty} \cap c\left(A_{1}\right) \subset\{\infty\}$ and $\overline{\mathbb{R}}=\mathbb{R} U\{\infty\}$ is regarded as the one point compactification of $\mathbb{R}$. PROOF. The equivalences (i) $\Leftrightarrow(i i) \Leftrightarrow(i i i)$ follow from Theorem 1.1. The equivalence (iv) $<=>(v)$ follows from results of section 3. We prove (i) $\Leftrightarrow\left(\right.$ iv) now. Let a $\epsilon \varrho\left(A_{F}\right)$ and $f \in M_{a} \cap D\left[J A_{F}\right]$. Since $M_{a} \subset D\left(A^{+}\right)$and $D[J A]=D\left[J A_{F}\right]$, (ii) implies $f \in D\left(A_{F}\right)$. It follows that $f=0$. This proves (iv). Suppose that (iv) holds and let $f \in D\left(A_{F}\right) \subset D\left(A^{+}\right)$. Since a $\in \varrho\left(A_{1}\right)$ we have $D\left(A^{+}\right)=D\left(A_{1}\right)+M_{a}$. Hence, there is a $g \in D\left(A_{1}\right)$ such that $f-g \in M_{a}$. As $D\left[J A_{F}\right] \subset D\left[J A_{1}\right]$, we have $f-g \in M_{a} \cap D\left[J A_{1}\right]=\{0\}$. Consequently, $f=g \in \mathbf{D}\left(A_{1}\right)$. This proves $\mathbf{D}\left(A_{F}\right) \subset \mathbf{D}\left(A_{1}\right)$. Therefore $A_{1}=A_{F}, i . e .,(i v) \Rightarrow(i)$. REMARK 2.2. It is easy to see that the definition of the Friedrichs extension of $A$ in the Krein space $k$ does not depend on the choice of the fundamental symmetry $J$. It is also true that $A_{F}=S_{F} J$, where $S=A J$ and $S_{F}$ is the Friedrichs extension of $S$ in the Hilbert space $(x,(\cdot)$,$) . Indeed, J S_{F}^{J}$ is a selfadjoint extension of $J S J=J A$ in $(K,(.,)$.$) and$ $\mathbf{D}\left[\mathrm{JS}_{\mathrm{F}^{J}}\right]=\mathrm{JD}\left[\mathrm{S}_{\mathrm{F}}\right]=\mathrm{JD}[\mathrm{S}]=\mathrm{D}[\mathrm{JSJ}]=\mathrm{D}[\mathrm{JA}]$.

Let $A$ be a positive operator in the Krein space $(K,[\cdot, \cdot])$. Then the operator $S=A J$ is a positive operator in the Hilbert space $(\boldsymbol{K},(., \cdot))$. Denote by $S_{K}$ the Krein extension of $S$ in $(K,(.)$,$) . The operator A_{K}:=S_{K} J$ is a positive selfadjoint extension of $A$ in $(K,[.]$.$) and we call$ it the krein extension of $A$ in the Krein space $k$.

In what follows we consider the set $R[A J]$ defined in the Hilbert space $(R,(\ldots))$. The set $R[A J]$ consists of all $\mathbf{f} \in \mathbf{K}$ for which there exists a sequence $\left(\phi_{\mathrm{n}}\right) \subset \mathbf{D}(\mathrm{A})$ such that $\mathrm{A} \phi_{\mathrm{n}} \rightarrow \mathrm{f}$ in $\mathbf{K}$ and

$$
\left[A\left(\phi_{n}-\phi_{m}\right), \phi_{n}-\phi_{m}\right] \rightarrow 0(n, m \rightarrow+\infty)
$$


Hence $R[A J]$ is independent of the choice of $J$.

REMARK 2.3. For the positive operator $A$ in the Krein space $(\mathbf{K},[.,]$.$) , the vector space \mathbf{R}(A)$ with the inner product

$$
\langle A f, A g\rangle:=[A f, g] \quad(f, g \in D(A))
$$

is a pre-Hilbert space. This space is contained continuously in $\mathbf{R}$ if and only if the operator $A$ is bounded. In order to see this, let $I$ denote the closure of $R(A)$ in $K$ and consider the selfadjoint positive operator $P:=A J \mid L$ in the Hilbert space $(L,(.,)$.$) . The operator A$ is bounded in $R$ if and only if $P$ is bounded in $L$. We have $\operatorname{ker}(P)=\{0\}$ and

$$
\langle\mathrm{f}, \mathrm{g}\rangle=\langle\mathrm{AJX}, \mathrm{AJY}\rangle:=\left[\mathrm{AJX}, \mathrm{JY}_{\mathrm{Y}}\right]=(\mathrm{AJX}, \mathrm{Y})=\left(\mathrm{f}, \mathrm{P}^{-1} \mathrm{~g}\right)
$$

for $f, g \in R(A)=R(P)$ and $x, y \in L$ such that $f=P x, g=P y$. The space $(R(A),<, \ldots)$ is contained continuously in $(K,(,)$, if and only if $\langle f, f\rangle \geq \alpha(f, f)$ ( $f \in R(A)$ ) for some $\alpha>0$, i.e., if and only if

$$
\left(f, P^{-1} f\right) \geq \alpha(f, f) \quad(f \in R(A)) .
$$

The last inequality holds if and only if $P$ is bounded. In this case the unique completion of $(R(A),<\ldots,>)$ is the Hilbert space $(R[A J],<,, .>)$. It is easy to see that $A$ coincides with the adjoint of the inclusion of $(\mathrm{R}[\mathrm{AJ}],<, \ldots\rangle)$ in $(\mathrm{K},[, \ldots])$. This is an alternative proof of Theorem 1 in $[d B]$ and the approach given here might be extended to other cases considered in [aB]. THEOREM 2.4. Let $A$ be a positive operator and let $A_{1}$ be a positive definitizable extension of $A$ in the Krein space $(K,[\cdot, \cdot])$. Denote by $E$ the spectral function of $A_{1}$. The following statements are equivalent:

(i) $\mathrm{A}_{1}=\mathrm{A}_{\mathrm{K}} . \quad\left(i_{1}\right) \quad \mathrm{D}\left(\mathrm{A}_{1}\right)=\left\{f \in \mathrm{K}: \mathrm{A}^{+} f \in R[\mathrm{AJ}]\right\}$.

(ii) $R\left(A_{1}\right)=R\left(A^{+}\right) \cap R[A J]$.

(iii) $R\left[A_{1} J\right]=R\left[A_{J}\right]$.

(iv) For some (and hence for all) a $\epsilon \varrho\left(A_{1}\right)$ we have

$$
\mathbf{M}_{\mathrm{a}} \cap \mathbf{R}\left[\mathrm{A}_{\left.1^{\top}\right]}=\{0\}\right.
$$


Suppose that $\operatorname{ker}\left(A_{1}\right)$ is orthogonally complemented in $(K,[., \cdot])$. Then the previous statements are equivalent to:

(v) For some (and hence for all) a $\epsilon \varrho\left(A_{1}\right)$ and for each $\phi \in M_{a}$ there exists $g \epsilon\{\phi\} \cup \operatorname{ker}\left(A_{1}\right)$ such that

$$
\int_{\mathbb{R}} 1 / t d\left[E_{t} \phi, g\right]=+\infty
$$

PROOF. The equivalences $(i)<=>\left(i_{1}\right) \Leftrightarrow>(i i) \Leftrightarrow($ iii)

follow from Theorem 1.3. The equivalence (iv) $\Leftrightarrow(v)$ follows from results of section 3 . We prove $(i)<=>$ (iv) now. Let a $\in \varrho\left(A_{K}\right)$

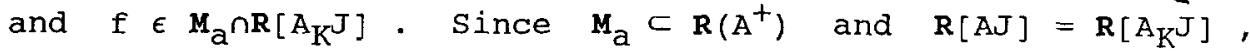
(ii) implies $f \in R\left(A_{K}\right)$. Let $f=A_{K} g$, $g \in D\left(A_{K}\right)$. Then $A^{+} f=a A_{K} g$, i.e., $f-a g \in M_{O} \subset D\left(A_{K}\right)$. Hence $f \in D\left(A_{K}\right)$ and, consequently $f=0$. This proves (iv). Suppose that (iv) holds and let $f \in \mathbf{N}_{-1} \cap R\left[S_{1}\right]$, where $S_{1}=A_{1} J$. As in the proof of Theorem 1.3, (iv) implies that $M_{0} \subset D\left(A_{1}\right)$. We have $f \in R\left(S^{*}\right)$ $=\mathbf{R}\left(A^{+}\right)$and $\boldsymbol{R}\left(A^{+}\right)=\mathbf{R}\left(A_{1}\right)+M_{a}$. Hence $f=g+h$, with $g \in \mathbf{R}\left(A_{1}\right), \quad h \in M_{a}$. It follows that $f-g \in M_{a} \cap R\left[A_{1} J\right]=\{0\}$. Consequently, $f=g \in R\left(A_{1}\right)=R\left(S_{1}\right)$. Let $f=S_{1} u$, with $u \in D\left(S_{1}\right)$. Then $S^{*} f=-S_{1} u$, i.e., $f+u \in N_{O} \subset D\left(S_{1}\right)$. Thus $f \in D\left(S_{1}\right)$ and $S_{1} f=-f$. Therefore $f=0$, i.e., $N_{-I} \cap R_{[}\left[S_{1}\right]$ $=\{0\}$. Theorem 1.3 yields $s_{1}=s_{K}$, and (iv) $\Rightarrow$ (i) is proved. REMARK 2.5. It follows from Theorem 2.3 that the definition of the Krein extension of $A$ in the krein space $\mathbf{x}$ does not depend on the special choice of the fundamental symmetry $J$. It is also true that $A_{K}=J B_{K}$, where $B=J A$ and $B_{K}$ is the Krein extension of $B$ in the Hilbert space $(K,(.)$,$) . Indeed,$ $J B_{K} J$ is a selfadjoint extension of $J B J=A J$ in $(K,(.)$,$) and$ $R\left[J_{K^{J}}\right]=J R\left[B_{K}\right]=J R[B]=J R[J A]=R[A J]$.

REMARK 2.6. Suppose that for the operator $A$ the form $[A f, g](f, g \in D(A))$ has a finite number of negative squares, $R(A-z I)$ is closed for at least one $z \epsilon \phi$ and that $A$ has $a$ finite defect $m_{0}$ (that is, there exists a selfadjoint extension $A_{1}$ of $A$ in $(K,[\cdot, \cdot])$ such that $\left.\operatorname{dim} D\left(A_{1}\right) / D(A)=m_{0}<+\infty\right)$. Then all selfadjoint extensions of $A$ in $(K,[, .]$.$) are defini-$ tizable. Particularly, if $A$ is a strictly positive operator in $(\mathrm{K},[. .]$.$) with a finite defect then all its selfadjoint exten-$ 
sions are definitizable. Moreover, each one of these selfadjoint extensions has an orthogonally complemented kernel (see [CL, Section 1.2]).

\section{A CHARACTERIZATION OF D[JA] FOR A DEFINITIZABLE A}

For a selfadjoint operator $S$ in the Hilbert space $(H,(\cdot, \cdot))$ we have $D[S]=D\left(|S|^{1 / 2}\right)$. Let $F$ denote the spectral function of $S$. Then the set $D[S]$ consists of all $f \in H$ such that $\int_{\mathbb{R}}|t| d\left(F_{t} f, f\right)<+\infty$. In Remark 3.3 and Theorem 3.4 of this section we give some analogous properties of the set $D[J A]$ with respect to the spectral function of a definitizable operator A.

Let $A$ be a definitizable operator in a Krein space $(\mathrm{K},[., \cdot])$ and let $\mathrm{E}$ denote the spectral function of $A$. Let $\Omega_{\infty}$ be a closed neighborhood of $\infty$ in $\mathbb{R}$ such that $\Omega_{\infty} \cap c(A)$ $c\{\infty\}$. Denote by $\Sigma_{\infty}$ the set of all $f \in \mathbb{K}$ such that

$$
\left|\int_{\Omega_{\infty}} t d\left[E_{t} f, f\right]\right|<+\infty .
$$

It follows from the properties of the spectral function $E$ that the set $I_{\infty}$ is independent of the choice of the neighborhood $\Omega_{\infty}$

PROPOSITION 3.1. Let $A$ be a boundedly invertible positive operator in the Krein space $(\mathrm{K},[., \cdot])$. Then $f \in D[J A]$ if and only if $\int_{\mathbb{R}} t d\left[E_{t} f, f\right]<+\infty$, i.e., $D[J A]=L_{\infty}$.

PROOF. We first prove that $\int_{\mathbb{R}} t d\left[E_{t} f, f\right]<+\infty$ for all $f \in D[J A]$. Since $J A$ is a positive operator in the Hilbert space $(K,(.)$,$) , we have that f \in D[J A]$ if and only if there exists a sequence $\left(\phi_{n}\right) \subset D(A)$ such that $\phi_{n} \rightarrow f$ and

$$
\left[\mathrm{A}\left(\phi_{\mathrm{n}}-\phi_{\mathrm{m}}\right), \phi_{\mathrm{n}}-\phi_{\mathrm{m}}\right] \rightarrow 0 \quad(\mathrm{n}, \mathrm{m} \rightarrow+\infty)
$$

Let $\Omega_{\mu}=(-\mu, \mu)$ for $\mu \in \mathbb{R}^{+}$. Then we have

$$
\left[A\left(\phi_{\mathrm{n}}-\phi_{\mathrm{m}}\right), \phi_{\mathrm{n}}-\phi_{\mathrm{m}}\right] \geq\left[\mathrm{AE}\left(\Omega_{\mu}\right)\left(\phi_{\mathrm{n}}-\phi_{\mathrm{m}}\right), \phi_{\mathrm{n}}-\phi_{\mathrm{m}}\right] .
$$


Curgus

The relation (3.1) implies that for each $\varepsilon>0$ there exists a natural number $N(\varepsilon)$ such that

$$
\left[\mathrm{AE}\left(\Omega_{\mu}\right)\left(\phi_{\mathrm{n}}-\phi_{\mathrm{m}}\right), \phi_{\mathrm{n}}-\phi_{\mathrm{m}}\right]<\varepsilon\left(\mathrm{n}, \mathrm{m} \geq \mathrm{N}(\varepsilon), \mu \in \mathbb{R}^{+}\right)
$$

As the operator $\operatorname{AE}\left(\Omega_{\mu}\right) \quad\left(\mu \in \mathbb{R}^{+}\right)$is bounded, letting $\mathrm{m} \rightarrow+\infty$ we get

$$
\left[A E\left(\Omega_{\mu}\right)\left(\phi_{\mathrm{n}}-f\right), \phi_{\mathrm{n}}-\mathrm{f}\right] \leq \varepsilon \quad\left(\mathrm{n} \geq \mathrm{N}(\varepsilon), \mu \in \mathbb{R}^{+}\right) .
$$

With $\mathrm{f}^{\langle\mu\rangle}:=\mathrm{E}\left(\Omega_{\mu}\right) \mathrm{f}, \mu \in \mathbb{R}^{+}$, we have

$$
\left[A E\left(\Omega_{\mu}\right)\left(\phi_{\mathrm{n}}-\mathrm{f}^{<\mu>}\right), \phi_{\mathrm{n}}-\mathrm{f}^{<\mu>}\right] \leq \varepsilon \quad\left(\mathrm{n} \geq \mathrm{N}(\varepsilon), \mu \in \mathbb{R}^{+}\right) \cdot(3.2)
$$

It is obvious that $\mathbf{R}(A)=\mathbf{K}$ and $\operatorname{ker}(A)=\{0\}$. Therefore

Theorem 6.1 (ii) in [L2] implies that $g=\int_{\mathbb{R}} d E_{t} g$ for all $g \in \mathrm{D}(\mathrm{A})$. Therefore $\lim _{\mu \rightarrow \infty} \mathrm{E}\left(\Omega_{\mu}^{\mathrm{C}}\right) \mathrm{g}=0$ for all $\mathrm{g} \in \mathrm{D}(\mathrm{A})$. Here $\Omega_{\mu}^{\mathrm{C}}=\overline{\mathbb{R}} \backslash \Omega_{\mu}$. Hence

$$
\begin{aligned}
& {\left[\mathrm{Ag}, \mathrm{E}\left(\Omega_{\mu}^{\mathrm{C}}\right) \mathrm{g}\right] \rightarrow 0 \quad(\mu \rightarrow+\infty, \mathrm{g} \in \mathrm{D}(\mathrm{A}))} \\
& \text { Since }\left(\phi_{n}\right) \subset D(A) \text {, for a fixed natural number } \\
& \mathrm{n}_{\mathrm{O}} \geq \mathrm{N}(\varepsilon) \text {, there exists } \mu_{0}=\mu_{0}\left(\mathrm{n}_{0}, \varepsilon\right)=\mu_{0}(\varepsilon) \text { such that } \\
& {\left[A \phi_{\mathrm{n}_{\mathrm{O}}}, E\left(\Omega_{\mu}^{\mathrm{C}}\right) \phi_{\mathrm{n}_{\mathrm{O}}}\right]<\varepsilon \quad\left(\mu \geq \mu_{\mathrm{O}}(\varepsilon)\right) .}
\end{aligned}
$$

Together with (3.2) this implies

$$
\begin{aligned}
& {\left[A\left(\phi_{\mathrm{n}_{0}}-\mathrm{f}^{\langle\mu\rangle}\right), \phi_{\mathrm{n}_{\mathrm{o}}}-\mathrm{f}^{\langle\mu\rangle}\right]=\left[\mathrm{AE}\left(\Omega_{\mu}^{\mathrm{C}}\right)\left(\phi_{\mathrm{n}_{\mathrm{o}}}-\mathrm{f}^{\langle\mu\rangle}\right), \phi_{\mathrm{n}_{\mathrm{o}}}-\mathrm{f}^{\langle\mu\rangle}\right]} \\
& +\left[A E\left(\Omega_{\mu}\right)\left(\phi_{\mathrm{n}_{0}}-\mathrm{f}^{\langle\mu\rangle}\right), \phi_{\mathrm{n}_{0}}-\mathrm{f}^{\langle\mu\rangle}\right] \\
& =\left[\operatorname{AE}\left(\Omega_{\mu}^{C}\right) \phi_{\mathrm{n}_{0}}, \phi_{\mathrm{n}_{\mathrm{o}}}\right]+\left[\operatorname{AE}\left(\Omega_{\mu}\right)\left(\phi_{\mathrm{n}_{\mathrm{o}}}-\mathrm{f}\right), \phi_{\mathrm{n}_{\mathrm{o}}}-\mathrm{f}\right] \\
& \leq \varepsilon+\varepsilon=2 \varepsilon \quad\left(\mu \geq \mu_{0}(\varepsilon)\right) \text {. }
\end{aligned}
$$

It follows from the last inequality that

$$
\left[\mathrm{A}\left(\mathrm{f}^{<\mu>}-\mathrm{f}^{\langle\tau\rangle}\right), \mathrm{f}^{<\mu>}-\mathrm{f}^{<\tau>}\right] \leq 8 \varepsilon \quad\left(\mu, \tau \geq \mu_{\mathrm{o}}(\varepsilon)\right) .
$$

since 


$$
\left[\mathrm{A}\left(\mathrm{f}^{\langle\mu\rangle}-\mathrm{f}^{\langle\tau\rangle}\right), \mathrm{f}^{\langle\mu\rangle}-\mathrm{f}^{\langle\tau\rangle}\right]=\int_{\mu} \mathrm{t} \mathrm{s} \tau \mathrm{d}\left[\mathrm{E}_{\mathrm{t}} \mathrm{f}, \mathrm{f}\right]
$$

for $\mu>T>0$, the inequality (3.3) yields $\int_{\mathbb{R}} t d\left[E_{t} f, f\right]$ $<+\infty$. Hence we have proved that $\mathbf{D}[\mathrm{JA}] \subset \mathrm{L}_{\infty}$.

Before we continue the proof of proposition 3.1 , we observe that if $\int_{\mathbb{R}} d E_{t} g$ converges in $\mathbf{k}$, then $\int_{\mathbb{R}} d E_{t g}=g$. Indeed, if we put $h=\int_{\mathbb{R}} d E_{t} g$, then

$$
A^{-1} h=\int_{\mathbb{R}} t^{-1} d_{t} h=\int_{\mathbb{R}} t^{-1} d_{E}\left(\int_{\mathbb{R}} d E_{\lambda} g\right)=\int_{\mathbb{R}} t^{-1} d_{t} g=A^{-1} g .
$$

Hence $\mathrm{g}=\mathrm{h}$.

Now we prove that $\mathrm{L}_{\infty} \subset \mathrm{D}[\mathrm{JA}]$. For $f \in \mathrm{I}_{\infty}, \mathrm{n} \geq \mathrm{m}$, and with the notation introduced above, we have

$$
\left[A\left(f^{<n>}-f^{<m>}\right), f^{<n>}-f^{<m>}\right]=\int_{\Omega_{n} \backslash \Omega_{m}} t d\left[E_{t} f, f\right] \rightarrow 0(n, m \rightarrow+\infty) \cdot(3.4)
$$

We note that $\left(f^{<n>}\right) \subset D(A)$. Since we suppose that $0 \in \varrho(A)$, we have

$$
[A g, g] \geq\left\|A^{-1}\right\|^{-1}\|g\|^{2} \quad(g \in D(A)) .
$$

This inequality and (3.4) imply that $\left(\mathrm{f}^{\langle\mathrm{n}\rangle}\right)$ is a cauchy sequence with respect to the norm $\|$.$\| in k$. It follows that $\int_{\mathbb{R}} d E_{t} f$ converges in $\mathbf{k}$. The preceding observation yields

$$
\int_{\mathbb{R}} \mathrm{dE}_{\mathrm{t}} \mathrm{f}=\mathrm{f}, \text { i.e., } \mathrm{f}^{<\mathrm{n}>} \rightarrow \mathrm{f} \quad(\mathrm{n} \rightarrow+\infty)
$$

The relations (3.4) and (3.5) imply that $f \in D[J A]$. The proposition is proved.

REMARK 3.2. It follows from the last part of the proof of Proposition 3.1 that

$$
\int_{\mathbb{R}} d E_{t} f=f \text { for all f } \epsilon \mathrm{D}[J A] \text {. }
$$


This is an improvement of Theorem 6.1 (ii) in [L2] for the operator A with $0 \in \varrho(A)$.

REMARK 3.3. The vector space $D[J A]$ with the inner product $\left((J A)^{1 / 2},,(J A)^{1 / 2} \cdot\right)$ is a Hilbert space and this inner product coincides on $D(A)$ with $[A .,$.$] . For f \in D[J A]$ and the sequence $\left(f^{<n>}\right) \subset D(A)$, defined as in the proof of proposition 3.1 , the relations (3.4) and (3.5) imply that $\mathrm{f}^{<\mathrm{n}\rangle} \rightarrow \mathrm{f}$ $(n \rightarrow+\infty)$ in the Hilbert space $\mathbf{D}[J A]$. Therefore, for every $f, g \in D[J A]$ we have

$$
\begin{aligned}
& \int_{\mathbb{R}} t d\left[E_{t} f, g\right]=\lim _{n \rightarrow \infty} \int_{\Omega_{n}} t d\left[E_{t} f, g\right]=\lim _{n \rightarrow \infty} \int_{\Omega_{n}} t d\left[E_{t} f^{<n>}, g^{<n>}\right] \\
& =\lim _{n \rightarrow \infty}\left[A f^{<n>}, g^{<n>}\right]=\lim _{n \rightarrow \infty}\left((J A)^{1 / 2} f^{<n>},(J A)^{1 / 2} g^{<n>}\right) \\
& =\left((J A)^{1 / 2} f,(J A)^{1 / 2} g\right) .
\end{aligned}
$$

THEOREM 3.4. Let $A$ be a definitizable operator.

Then

$$
D[J A]=\mathbf{L}_{\infty}
$$

PROOF. Let $\Omega_{\infty} \subset \overline{\mathbb{R}}$ be a closed neighborhood of $\infty$ which does not contain zero and $\Omega_{\infty} n c(\mathrm{~A}) \subset\{\infty\}$. Put $\mathbf{k}_{\infty}:=$ $E\left(\Omega_{\infty}\right) \mathbf{K}$. Let $J_{O}$ be a fundamental symmetry on $\mathrm{K}$ which commutes with $E\left(\Omega_{\infty}\right)$. Then $J_{0} \mid \mathbf{K}_{\infty}$ is a fundamental symmetry on $\mathbf{k}_{\infty}$. The restriction $A_{\infty}:=A \mid \mathbf{K}_{\infty}$ is a boundedly invertible operator. If the operator $A$ has a definitizing polynomial of even degree, then $\left(K_{\infty},|[\cdot, \cdot]|\right)$ is a Hilbert space and the equality $\mathrm{D}\left[\mathrm{J}_{\mathrm{O}} \mathrm{A}_{\infty}\right]=\mathbf{L}_{\infty}$ follows easily.

If the operator $A$ has a definitizing polynomial of odd degree, than $A_{\infty}$ is a boundedly invertible positive or negative operator in the krein space $\left(\mathbf{k}_{\infty},[\cdot, \cdot]\right)$. By $\mathbf{k}_{\infty}^{\perp}$ we denote the orthogonal complement of $k_{\infty}$ in $(K,[, \ldots])$. The decomposition $\mathbf{K}=\mathbf{K}_{\infty}^{\perp}[\dot{+}] \mathbf{k}_{\infty}$, reduces the operators $J_{O} A,\left|J_{O} A\right|$ and the restriction $\left|J_{O} A\right| \mid \mathbf{K}_{\infty}^{\perp}$ is a bounded operator. Here $\left|J_{O} A\right|$ denotes the absolute value of the selfadjoint operator $J_{O} A$ in the Hilbert space $\left(K,\left[J_{0} ..\right]\right)$. The same decomposition 
reduces the operator $\left(\left|J_{0} A\right|+I\right)^{1 / 2}$ and we conclude that

$$
\mathrm{D}\left(\left(\left|J_{0} \mathrm{~A}\right|+I\right)^{1 / 2}\right)=\mathbf{K}_{\infty}^{\perp}[\dot{+}] \mathrm{D}\left(\left|\mathrm{J}_{\mathrm{O}} \mathrm{A}\right|^{1 / 2}\right) .
$$

It follows from [C, Remark 1.4] that

$$
\mathrm{D}\left[\mathrm{J}_{\mathrm{O}} \mathrm{A}\right]=\mathrm{D}\left(\left(\left|\mathrm{J}_{\mathrm{O}} \mathrm{A}\right|+\mathrm{I}\right)^{1 / 2}\right) \text { and } \mathrm{D}\left[\mathrm{J}_{\mathrm{O}} \mathrm{A}_{\infty}\right]=\mathrm{D}\left(\left|\mathrm{J}_{\mathrm{O}} \mathrm{A}_{\infty}\right|^{1 / 2}\right) \text {. }
$$

Hence

$$
\mathrm{D}\left[\mathrm{J}_{0} \mathrm{~A}\right]=\mathbf{K}_{\infty}^{\perp}[\dot{+}] \mathbf{D}\left[\mathrm{J}_{0} \mathrm{~A}_{\infty}\right]
$$

Proposition 3.1 implies that $D\left[\mathcal{J}_{0} A_{\infty}\right]$ consists of ali $f \epsilon k$ for which $\left|\int_{\Omega_{\infty}} t d\left[E_{t} f, f\right]\right|<+\infty$. It follows from this and (3.6)

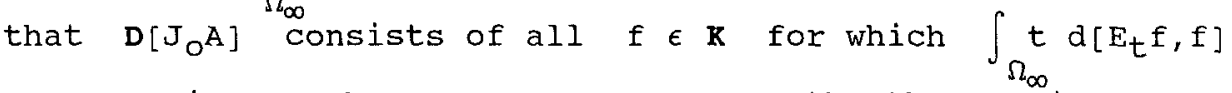
$<+\infty$. Since we have $D\left[J_{0} A\right]=D[J A]$, the theorem is proved. REMARK 3.5. Remark 3.2 and the equality (3.6) imply that for every $f \in D[J A]$ we have

$$
E\left(\Omega_{\infty}\right) f=\int_{\Omega_{\infty}} d E_{t} f .
$$

Note that if $\infty$ is a regular critical point of the definitizable operator $A$ the equality (3.7) holds for every $f \in K$.

If $S$ is a positive selfadjoint operator in a Hilbert space $(H,(.)$,$) , it follows from Lemma 1.2$ that $R[S]$ consists of all $f \in \mathbf{H}$ for which $\int_{\mathbb{R}} 1 / t d\left(F_{t} f, f\right)<+\infty$. In the following proposition we show an analogous property of $\mathbf{R}[\mathrm{AJ}]$ with respect to the spectral function $E$ of the positive definitizable operator A.

Denote by $L_{O}$ the set of all $f \in R$ such that

$$
\int_{\mathbb{R}} 1 / t d\left[E_{t} f, g\right]<+\infty \quad \text { for all } g \in\{f\} U \operatorname{ker}(A) \text {. }
$$

PROPOSITION 3.6. Let $A$ be a positive definitizable operator in $(K,[., \cdot])$ and suppose that $\operatorname{ker}(A)$ is orthogonally complemented in $\mathbf{K}$. Then we have $\mathbf{R}[A J]=\mathbf{L}_{\circ}$.

PROOF. Let $\mathbf{L}$ be the closure of $\mathbf{R}(A)$ in $\mathbf{K}$. Then 
$\mathbf{K}=\operatorname{ker}(A)[\dot{+}] \mathbf{L},(L,[, \ldots])$ is a Krein space and $A / L$ is a positive definitizable operator in $I$ such that $\operatorname{ker}(A \mid L)=\{0\}$. Let $J_{0}$ be a fundamental symmetry on $I$ and let $Q$ be the orthogonal projector onto $L$ in $K$. Obviously $Q E / L$ is a spectral function of $A \mid L$ and $R\left[(A \mid L) J_{0}\right]=D\left[J_{O}(A \mid L)^{-1}\right]$. It follows from the properties of the spectral function of $A / L$ (see [L2, II.3]) and Theorem 3.4 that $R\left[(A \mid L) J_{0}\right]$ is the set of all $f \in I$ for which

$$
\int_{\mathbb{R}} 1 / t d\left[E_{t} f, f\right]<+\infty
$$

The last set coincides with $\mathrm{L}_{0}$. Indeed, it is obvious that for every $f \in L$ and $g \in \operatorname{ker}(A)$ we have $\int_{\mathbb{R}} 1 / t d\left[E_{t} f, g\right]=0$.

Hence for $f \in I$ the relation (3.8) yields $f \epsilon L_{O}$. Conversely, for $f \epsilon I_{O}$ and for all $g \epsilon \operatorname{ker}(A)$ we have

$$
\infty+>\left|\int_{\mathbb{R}} 1 / t d\left[E_{t} f, g\right]\right|=\left|\int_{\mathbb{R}} 1 / t d\left[E_{t}(I-Q) f, g\right]\right| .
$$

This implies that $[(I-Q) f, g]=0$ for all $g \in \operatorname{ker}(A), i . e$. , f $\epsilon \mathrm{L}$ and (3.8) holds.

Since $\mathbf{R}[A J]=\mathbf{R}\left[(A \mid L) J_{0}\right]$, the proposition is proved.

The following proposition is an improvement of Theorem 6.1 (ii) in [L2] for the operator A with the orthogonally complemented kernel.

PROPOSITION 3.7. Let $A$ be as in proposition 3.6 . Then we have

$$
f=\int_{\mathbb{R}} d E_{t} f \quad(f \in D[J A] \cap R[A J])
$$

PROOF. It follows from Remark 3.2 and the properties of the spectral function $E$ that for a bounded positive operator $A$ in $K$ such that $\operatorname{ker}(A)=\{0\}$ we have $f=\int_{\mathbb{R}} d E_{t} f$ for all $f \epsilon R[A J]$. It is obvious that the same equality holds true with an orthogonally complemented $\operatorname{ker}(A)$. For an unbounded $A$ and $f \epsilon R[A J] \cap D[J A]$ put $f_{0}=E\left(\Omega_{1}\right), f_{\infty}=f-f_{0}, k_{0}=E\left(\Omega_{1}\right) K$ and $\mathbf{K}_{\infty}=E\left(\mathbb{R} \backslash \Omega_{I}\right) \mathbf{K}$. Let $\mathrm{J}$ be a fundamental symmetry on $\mathbf{K}$ which 
Commutes with $E\left(\Omega_{1}\right)$. Then $f_{0} \in R\left[\left(A \mid K_{0}\right)\left(J \mid K_{0}\right)\right]$,

$f_{\infty} \in D\left[\left(J \mid k_{\infty}\right)\left(A \mid k_{\infty}\right)\right]$ and the preceding remark yields

$$
\mathrm{f}_{\mathrm{o}}=\int_{\Omega_{1}} \mathrm{~d} \mathrm{E}_{\mathrm{t}} \mathrm{f}, \quad \mathrm{f}_{\infty}=\int_{\mathbb{R} \backslash \Omega_{1}} \mathrm{dE}_{\mathrm{t}^{\mathrm{f}}} .
$$

The proposition follows from the last two equalities.

\section{REFERENCES}

[AS] Alonso, A., Simon, B.: The Birman-Krein-Vishik theory of self-adjoint extensions of semibounded operators. J. Operator Theory 4 (1980), 251-270.

[AN] Ando, T., Nishio, K.: Positive selfadjoint extensions of positive symmetric operators. Tohoku Math. Journ. $22(1970), 65-75$.

[AI] Azizov, T.Ya., Iokhvidov, I.S.: Foundations of the theory of linear operators in spaces with indefinite metric. (Russian.) Nauka, Moscow, 1986.

[Bi] Birman, M.S.: On the self adjoint extensions of positive definite operators. (Russian.) Math. Sb. 38 (1956), 431-450.

[Bo] Bognár, J.: Indefinite inner product spaces. SpringerVerlag, Berlin / Heidelberg / New York, 1974.

[dB] de Branges, L.: Complementation in Krein spaces. Trans. Amer. Math. Soc. 305 (1988), 277-291.

[CS] Coddington, E.A., de Snoo H.S.V.: Positive selfadjoint extensions of positive symmetric subspaces. Math. Z. $159(1978), 203-214$.

[C] Curgus, B.: on the regularity of the critical point infinity of definitizable operators. J. Integral Equations Operator Theory 8 (1985), 462-488.

[CL] curgus, B., Langer, H.: A Krein space approach to symmetric ordinary differential operators with an indefinite weight function. To appear in $\mathrm{J}$. Differential Equations.

[F] Faris, W.G.: Self-adjoint operators. Lecture Notes in Mathematics 433, springer-Verlag, Berlin, 1975.

[J] Jonas, P.: on the functional calculus and the spectral function for definitizable operators in Krein spaces. Beitrage Anal. 16 (1981), 121-135.

[JL] Jonas, P., Langer, H.: Some questiones in the perturbation theory of $\mathrm{J}$-nonnegative operators in Krein spaces. Math. Nachr. 114 (1983), 205-226.

[Ka] Kato, T.: Perturbation theory for linear operators. 
[Kr] Krein, M.G.: The theory of self-adjoint extensions of semi-bounded Hermitian transformations and its applications. I (Russian.) Mat. Sb. (N.S.) 20 (62) (1947), 431-495.

[L1] Langer, H.: Verallgemainerte Resolventen eines Jnichtnegativen operators mit endlichem Defekt. J. Functional Analysis 8 (1971), 287-320.

[L2] Langer, H.: Spectral functions of definitizable operators in Krein spaces. Functional analysis, Proceedings of a conference held at Dubrovnik, Yugoslavia, November 2-14, 1981. Lecture Notes in Mathematics 948, Springer-Verlag, Berlin / Heidelberg / New York, 1982, 1-46.

[S] Skau, C.F.: Positive self-adjoint extensions of operators affiliated with a von Neumann algebra. Math. Scand. 44 (1979), 171-195.

[V] Vishik, M.: On general boundary conditions for ellyptic differential equations. (Russian.) Trudy Moskov. Mat. obsc. 1 (1952), 187-246.

[W] Weidmann, J.: Linear operators in Hilbert spaces. Springer-Verlag, New York / Heidelberg / Berlin, 1980.

Department of Mathematics Western Washington University

Bellingham, Washington 98225

U. S. A.

Submitted: December 19, 1988 